\title{
Influence de la mammite sur les propriétés technologiques du lait et sur la qualité des produits laitiers
}

\author{
par \\ G. WAES et M. Van BELLEGHEM \\ Station Laitière de l'Etat, Melle (Belgique)
}

\section{I. - INTRODUCTION}

L'augmentation considérable de la teneur en cellules et plus particulièrement du nombre des leucocytes du lait est à présent généralement admise comme un critère très valable pour le dépistage des maladies de la mamelle chez le bétail laitier.

Les désordres de la sécrétion de la mamelle, causés ou non par une infection, entraînent aussi cette conséquence, que la composition et les propriétés physiques du lait peuvent être modifiées.

La question qui se pose à ce propos est de savoir si, partant d'un tel lait, ou d'un lait de mélange contenant un certain pourcentage de lait de mammite, on peut fabriquer des produits laitiers de qualité ou s'il est nécessaire ou non d'adapter les procédés de fabrication.

Abstraction faite des aspects hygiéniques, un des plus graves problèmes posés par la mammite et plus spécialement par la lutte contre cette maladie est la présence d'antibiotiques dans le lait. Bien plus encore que par la modification de composition, laquelle est amortie par le mélange au lait normal, la présence de concentrations même relativement faibles d'antibiotiques peut troubler sérieusement certains procédés technologiques. Selon Pette (64), le préjudice déjà causé à l'industrie laitière par l'emploi intensif d'antibiotiques serait bien plus considérable que celui éprouvé du fait du lait de mammite.

La bibliographie fournit d'abondantes données sur les modifications de composition du lait imputables aux inflammations de la mamelle, les opinions étant cependant parfois assez divergentes à l'endroit de certains éléments. On est bien moins informé des conséquences sur le plan technologique et de l'influence exercée sur la qualité des produits finis. 


\section{II. - INFLUENCE DE LA MAMMITE SUR LA COMPOSITION DU LAIT}

La composition du lait normal subit déjà, en soi, de nombreuses influences telles que celles de la saison, de la race, de facteurs héréditaires et individuels, de l'alimentation, des soins, etc. Les teneurs en éléments divers varient moins, selon la règle de Wiegner, dans la mesure où ces éléments sont mieux dispersés dans le lait. Il s'ensuit que la fraction la plus variable serait la teneur en matières grasses, puis viendraient la fraction de caséine et les autres protéines du lait. Le lactose et les sels figurent à l'extrémité de la série.

Selon le degré de gravité de la mammite, on obtient d'après Pette (64), un lait plus ou moins modifié. Le changement de composition peut varier d'une modification à peine perceptible à une modification aisément visible, avec toute une échelle de possibilités intermédiaires.

Les modifications de la composition du lait seraient la conséquence du fait que la transformation d'éléments du sang en éléments normaux du lait est troublée (77). Selon Bortree, Caroll et Schalm (7) une perméabilité capillaire accrue au cours de l'inflammation entrâ̂nerait une admission plus rapide que la normale de protéines du sang dans le lait et Nicolet (57) parle aussi d'une perméabilité accrue de l'épithélium des glandes à la fraction de sérum du sang. La composition du lait pourrait, de ce fait, se rapprocher plus ou moins de celle du sang.

\section{Teneur en matières grasses et extrait sec dégraissé}

La littérature fournit des opinions divergentes quant à l'influence de l'inflammation de la mamelle sur la teneur en matières grasses du lait.

Dans du lait de quartiers, Seelemann (79), de même que Rowland et ses collaborateurs (73) font état d'une diminution de la teneur en matières grasses et de l'extrait sec dégraissé dans les cas de mammite. O'Donovan et ses collaborateurs (62) ont constaté dans des échantillons de quartiers de mamelle infectés de Staph. pyogènes, pour une période de lactation entière au cours de laquelle les quartiers malades furent traités, une baisse de 12 p. 100 de la teneur en matières grasses et de 11 p. 100 de la teneur en extrait sec dégraissé comparativement aux quartiers sains. Rowland et ses collaborateurs (73) ont constaté aussi une faible diminution du pourcentage d'extrait sec dégraissé dans le lait de quartiers infectés par Staph. pyogènes. La diminution était de $0,19 \pm 0,03 \mathrm{p}$. 100 pour les matières grasses et de $0,20 \pm 0,02$ pour l'extrait sec dégraissé. 
Waite et Blackburn (89) n'ont trouvé aucun rapport entre le nombre de cellules et la teneur en matières grasses ; par contre, ils ont observé un rapport négatif entre la teneur en extrait sec dégraissé et le nombre de cellules dans le lait de vaches individuelles. Une diminution de l'E.S.D. en cas de forte teneur en cellules a été observée par Alexander et Leech (1). Natzke et ses collaborateurs (55) ont également constaté une baisse de la teneur en extrait sec dégraissé du lait provenant de quartiers à réaction CMT positive comparés aux quartiers négatifs des mêmes animaux. Suivant Rook (71), la modification des matières grasses du lait de quartiers consécutive à la mammite est irrégulière, tandis que la modification de la teneur en extrait sec dégraissé dépend de la manière dont la diminution de lactose et de caséine est compensée par une plus forte teneur en protéines de sérum sanguin. Selon Teute (87), la teneur en matières grasses dans le lait de quartiers augmenterait, tant dans la mammite chronique que dans la mammite aiguë, de normalement 2,4 p. 100 à 3,1 p. 100 . Il nous semble que ces pourcentages sont plutôt faibles.

\section{Teneur en protéines}

\subsection{Protéines totales}

Beaucoup d'auteurs estiment que la teneur en protéines totales du lait provenant de vaches atteintes de mammite est plus élevée $(3,33,38,54,84)$. Teute $(87)$ a pu constater que le pourcentage relatif augmentait de 7,5 p. 100 dans le cas de la mammite chronique et de 14 p. 100 dans le cas de la mammite aiguë dans du lait de quartiers. Keis (28) a relevé une corrélation positive entre le degré d'inflammation du pis et la teneur en protéines totales dans du lait du bidon.

D'après Kästli (25) et Waite et Blackburn (90) la teneur en protéines totales dans du lait de quartiers resterait à peu près égale et Heeschen (19) aussi n'a découvert aucune relation entre la teneur en protéines totales dans du lait de quartiers (déterminée par la méthode au noir amido) et la présence de mammites ou de troubles de la sécrétion ; selon ce dernier auteur, la teneur en protéines totales peut demeurer inchangée, augmenter ou diminuer. Une baisse de la teneur en protéines totales du lait de quartiers dans diverses formes d'inflammation du pis a aussi été signalée par Renk et Teute (69).

\subsection{Caséine}

La littérature n'est pas plus concordante à propos de la modification de la teneur en caséine.

D'après Lerche (41), une baisse de la teneur en caséine du lait de quartiers est observée dans la plupart des cas de mammite, que celle-ci soit infectieuse ou non. Cette opinion est partagée par Nani et Redaelli (54), Kästli (25) et Renk et Teute (69). Kisza et Sobina (38) font état d'une diminution de la fraction de caséine par rapport aux autres substances azotées dans le lait de vaches prises indivi- 
duellement. Waite et Blackburn (89) ont relevé une baisse de la teneur en caséine du lait d'animaux pris individuellement quand la teneur en cellules dépassait \pm 1 million au $\mathrm{ml}$ ou quand ce lait contenait plus de 600000 cellules polynucléaires au ml. Ils ont fait les mêmes observations sur du lait de quartiers provenant d'un animal souffrant de la mammite subclinique (90), Dans les cas graves d'inflammation du pis ou dans la forme aiguë, la teneur en caséine du lait de quartiers retomberait, selon Teute (87) à 92,39 p. 100 de la teneur normale ; pour la mammite chronique elle s'élèverait, par contre, jusqu'à 105,43 p. 100 . Keis (28) et Kiermeier et Keis (33) ont relevé aussi une augmentation de la teneur en caséine. Ils ont même constaté une corrélation positive entre le degré d'inflammation du pis et la teneur en caséine du lait, ceci, tant pour le lait d'animaux pris individuellement que pour des échantillons prélevés au bidon.

\subsection{Protéines du sérum}

Une augmentation de Ia teneur en protéines du sérum du lait de vaches souffrant d'une maladie de la mamelle est quasi généralement admise $(3,19,33,38,40,57,71,84)$. Suivant Hauke (18), il se produit une diminution des fractions de protéines synthétisées dans la mamelle et un transfert accru d'éléments protéiques du sérum sanguin.

Cette modification de la fraction protéique du sérum lactique se manifesterait autant dans les maladies infectieuses de la mamelle que dans la mammite non infectieuse. Kästli (25) et Bortree, Caroll et Schalm (7) font, eux aussi, état d'une diminution de lactalbumine et de lactoglobuline et d'une augmentation de l'albumine et de la globuline du sang.

Selon Heeschen (19) l'augmentation de la teneur en protéines du sérum est perceptible dans le lait de quartiers, mais ne peut plus être distinguée dans le lait de vaches individuelles ou dans le lait de mélange.

$\mathrm{Au}$ début de l'inflammation de la mamelle, il se produit, selon Bortree et ses collaborateurs (7), une forte augmentation du pourcentage relatif d'albumine de sérum sanguin dans le sérum lactique tandis que l'augmentation d'immuno-globuline se produirait plus lentement. Suivant Hauke (18), ceci est en corrélation avec le poids moléculaire des protéines du plasma sanguin : étant relativement petites, les molécules d'albumine sérique franchiraient plus rapidement les capillaires à perméabilité accrue, les molécules d'immunoglobuline ne pouvant passer qu'après, du fait de leur plus grande taille. Dans le lait de quartiers provenant de vaches qui souffraient de mammite streptococcique chronique, Teute (87) à constaté une forte augmentation de la teneur en globuline tandis que celle de l'albumine restait au niveau normal. Ce même auteur fait état d'une forte augmentation de la teneur de ces deux espèces de protéines dans les cas aigus. La teneur en protéines du sérum du lait de 
vaches prises individuellement peut s'élever, selon Kisza et Sobina (38), jusqu'à 1 p. 100 dans l'inflammation chronique de la mamelle et jusqu'à 2,5 p. 100 dans la mammite aiguë.

\section{Teneur en lactose}

Il est généralement admis que la teneur en lactose du lait d'animaux aux mamelles malades est inférieure à la normale $(3,25,38$, $39,40,64,71,84,89,90)$. Il va de soi que cette baisse est la plus nette dans les échantillons prélevés aux quartiers de mamelle. Keis (28) a cependant pu constater aussi dans des échantillons prélevés au bidon une corrélation statistique négative, à vrai dire moins prononcée, mais tout de même confirmée, entre le degré d'inflammation du pis, déterminé par l'épreuve de Schalm modifiée, et la teneur en lactose du lait. Filipovitch et Filipovitch (15) n'ont, en revanche, découvert aucun rapport entre la teneur en lactose et la mammite dans le lait de vaches prises individuellement, ils ont même constaté dans de nombreux cas une teneur en lactose normale du lait chez des animaux atteints d'inflammation aiguë de la mamelle. Teute (87) a même relevé une augmentation de la teneur en lactose dans du lait de quartiers d'animaux souffrant de mammite chronique, mais il relève aussi une baisse dans les cas aigus.

\section{Teneur en sels}

\subsection{Chlorure.}

En ce qui concerne les constituants minéraux du lait, l'augmentation de la teneur en chlorure est particulièrement frappante dans les inflammations du pis. La littérature est aussi pratiquement unanime à ce sujet $(3,4,23,35,38,39,41,61,64,66,71,78,79,80)$. Selon Ashworth et Blosser (3), il existe un rapport direct entre la réaction CMT et la teneur en chlorure des échantillons prélevés au quartier. Kisza et ses collaborateurs $(35,38,39)$ estiment que, pour ce qui concerne l'inflammation du pis, l'indice chlore-sucre $\left(\frac{\text { chlore }}{\text { lactose }} \times 100\right)$ est un critère d'appréciation de l'état de santé des animaux. Cet indice est normalement d'environ 2. Les auteurs précités ont trouvé des valeurs allant de 3 à 6 chez des animaux atteints de mammite chronique, mais non clinique, et ont enregistré des indices chloresucre supérieurs à 10 dans l'inflammation aiguë. Kästli (25) fait, lui aussi, état d'une hausse de l'indice chlore-sucre en cas de mammite.

D'après Baumgartner et Walser (4) l'augmentation de la conductivité du lait de mammite par augmentation de la teneur en $\mathrm{NaCl}$ peut se mesurer dans les échantillons prélevés aux quartiers, mais pas dans ceux prélevés aux bidons. 
Filipovitch et Filipovitch (15) estiment qu'une forte teneur en $\mathrm{Cl}$ n'est pas nécessairement révélatrice de mammite. Ils ont trouvé fréquemment une teneur en chlorure normale $(0,07-0,14$ p. 100) dans du lait provenant d'animaux atteints de mammite aiguë.

\subsection{Autres substances minérales.}

Redaelli et Nani (66) ont examiné le lait des quartiers infectés et celui des quartiers sains provenant des mêmes vaches. La teneur en $\mathrm{Na}$ du lait des quartiers infectés avait augmenté et les teneurs en $\mathrm{K}$ et en $\mathrm{Ca}$ avaient diminué. Quant à la teneur en $\mathrm{Mg}$, ils ne relevèrent aucun écart significatif entre les quartiers infectés et les quartiers sains. Imamura et ses collaborateurs (23) ont, eux aussi, constaté une baisse des teneurs en $\mathrm{Ca}$ et en $\mathrm{K}$ de 8 échantillons de lait de mammite provenant des vaches prises individuellement, les teneurs en $\mathrm{P}$ et en Mo avaient aussi baissé et la teneur en $\mathrm{Na}$ augmenté. Kisza et ses collaborateurs (35) ont observé les baisses suivantes dans le lait d'animaux très malades : $\mathrm{Ca}:-36 \mathrm{p} .100$, P : -32 p. $100, \mathrm{Mg}:-20$ p. $100, \mathrm{~K}:-44$ p. 100 . La teneur en $\mathrm{Na}$ serait toutefois de 5,5 à 7 fois plus élevée que dans le lait normal.

Oberosler et ses collaborateurs (60) ont examiné l'ultrafiltrat d'échantillons de lait provenant de quartiers sains et de quartiers infectés de $S$. agalactiae. La teneur en phosphore total et en phosphore anorganique du lait provenant de quartiers infectés était plus élevée que celle du lait des quartiers sains tandis que la teneur en phosphore soluble dans l'acide était plus élevée dans l'ultrafiltrat du lait provenant des quartiers sains. Kisza, Karwowicz et Sobina (35) mentionnent, surtout, une diminution du phosphore minéral, estérifié et lipide.

\section{Teneur en vitamines}

L'impression que diverses vitamines ne sont présentes qu'en quantités moindres dans le lait de mammite n'est pas toujours confirmée par les recherches les plus récentes.

Davis et Mc Clement (10), qui ont examiné le lait de vaches prises individuellement, font état d'une diminution de la teneur en aneurine, en lactoflavine et en acide ascorbique du lait d'animaux au pis malade. Nani et Defranceschi (53) ont examiné le lait de 20 vaches au cours de différentes phases d'une infection par $S$. agalactiae. La teneur en toutes vitamines du complexe B augmentait au cours de la phase clinique. Marmori et ses collaborateurs (44) n'ont relevé aucun écart significatif entre les teneurs en carotène et en vitamine A des laits provenant les uns de quartiers sains et les autres des quartiers correspondants infecté de $S$. agalactiae. Ils ont constaté une légère augmentation de la teneur en vitamine A libre dans le lait de mammite (45). Selon Owen (63), il se produit une baisse de la teneur en vitamine A sous la forme d'ester et une hausse 
de la teneur sous la forme d'alcool, ceci du fait d'une estérification défectueuse dans la mamelle.

\section{Enzymes}

Bon nombre d'auteurs $(2,9,25,42,61,78,80)$ admettent que l'activité de catalase augmente dans le lait de mammite et que cette augmentation serait caractéristique de l'inflammation du pis. Müllen (52) et Keis (28) ont constaté dans le lait de mammite une augmentation de la phosphatase acide et le second auteur a relevé un rapport direct entre le degré d'inflammation du pis (déterminé par une épreuve de Schalm modifiée) et la teneur en phosphatase acide (exprimée en $\mu \mathrm{g}$ de phénol) du lait de vaches prises individuellement. Selon Kästli (25), la phosphatase acide est d'origine bactérienne et surtout les staphylocoques en seraient responsables. Un accroissement de l'activité de phosphatase est mentionné également par Arima (2). Owen (63) fait état d'une relation entre le degré d'infection et la teneur en phosphatase alcaline.

Wartenberg (91) rapporte que l'activité de l'amylase est nettement plus forte dans le lait provenant d'animaux atteints de mammite aiguë.

Nilsson (59) a trouvé dans le lait de mammite une teneur en oxydase de xanthine légèrement accrue. Selon cet auteur, (58), l'accroissement d'activité de l'oxydase de la xanthine serait en corrélation avec l'accroissement du pouvoir de réduction qu'elle a constaté dans le lait de quartiers infectés. Kiermeier et Graf zu SolmBaruth (30) ont observé un accroissement de l'activité de déhydrase de la xanthine couplé à l'augmentation de la teneur en cellules (déterminée par l'épreuve de Schalm).

Selon Ludecke (42), l'activité de l'arylestérase dans le lait de quartiers augmenterait en corrélation avec le degré de netteté de la réaction CMT. Marquardt et Forster (46) ont relevé dans du lait de quartiers à valeurs élevées de teneur en chlore, d'activité de catalase et de réaction CMT, une activité d'A-estérase de 10 à 12 fois plus forte que celle de lait normal.

Selon Guthrie et Herrington (16), qui examinaient le lait de vaches prises individuellement, la présence de mammite serait un facteur important de l'activité de lipase et du rancissement du lait.

\section{Conclusion}

On peut conclure de l'examen global des opinions relatives à l'influence de l'inflammation de la mamelle sur la composition du lait que celles-ci ne sont pas toujours concordantes. Ceci n'a rien d'étonnant, étant donné que le lait de mammite peut dévier de peu 
jusqu'à très fort du lait normal et que l'on fait usage de critères différents pour déterminer si l'on se trouve ou non en présence de lait de mammite.

De plus, les déterminations de certaines substances ne sont pas toujours faites par les mêmes méthodes.

Les tendances générales des opinions concernant les modifications de la composition du lait par les mammites se reflètent dans le tableau ci-dessous selon Kästli (25).

\section{Modifications du lait en cas de maladies de la mamelle}

Poids spécifique et matière séche $\ldots \ldots \ldots \ldots \ldots \ldots \ldots \ldots+$

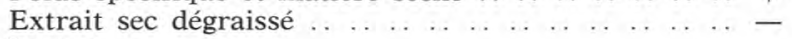

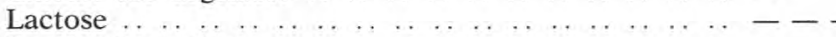

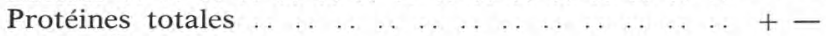

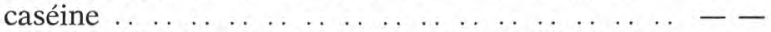

bêta-lactalbumine et bêta-lactoglobuline ... . . -

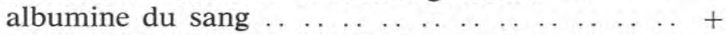

gamma-globuline (globuline du sang) ....... $\ldots+++$

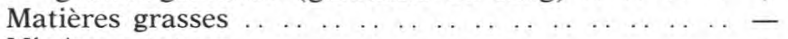

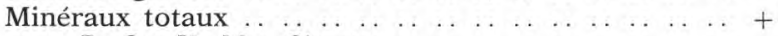

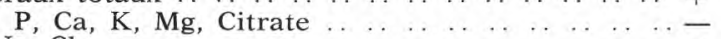



S $\ldots \begin{array}{llllllllllllllllllll} & \ldots & \ldots & \ldots & \ldots & \ldots & \ldots & \ldots & \ldots & \ldots & \ldots & \ldots & \ldots & \ldots & \ldots & \ldots & \ldots & +\end{array}$

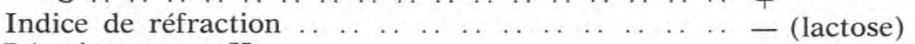

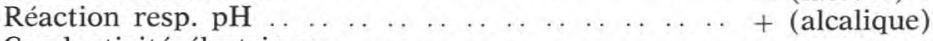

Conductivité électrique $\ldots \begin{array}{lllllllllllll} & \ldots & \ldots & \ldots & \ldots & \ldots & \ldots & \ldots & \ldots & +\end{array}$

Indice de chlore-sucre (selon Koestler) $\frac{\mathrm{CI} \times 100}{\text { lactose }} \cdots++$ (normale 2,5)

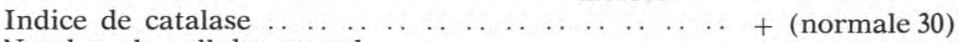

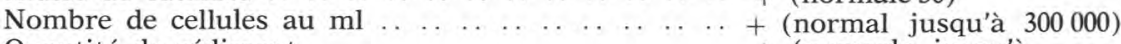

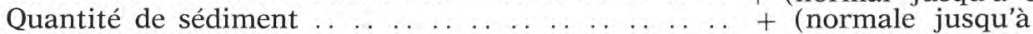

Epreuve de Whiteside ou épreuve de Schalm ... . . . +

$0,3$ p. 1000$)$

Aptitude à la coagulation ... . . . . . . . . . . . . . . . . .

Explication des symboles

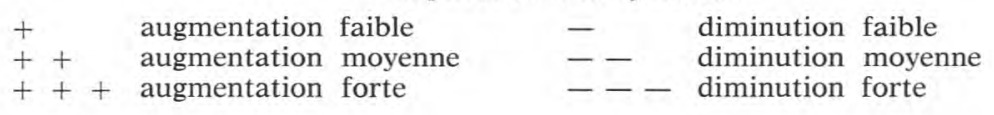

\section{III. - LE LAIT DE MAMMITE ET SES PROPRIETES TECHNOLOGIQUES}

L'aptitude du lait à constituer un milieu nutritif pour les ferments lactiques, joue un rôle considérable dans la fabrication de nombreux produits laitiers. La fabrication du fromage exige l'emploi 
d'un lait bien coagulable. La stabilité thermique est importante dans la fabrication d'autres produits laitiers.

\section{Aptitude à l'acidification}

Les propriétés du lait de mammite influent sur les possibilités d'utilisation technologique tant du point de vue du nombre de germes initial que de celui de l'aptitude à offrir un milieu nutritif aux ferments lactiques.

Kästli et Graber (26) ont démontré que les bactéries provenant de mamelles catarrheuses sont freinées dans leur développement dans le lait de mammite. Une nette diminution du nombre de germes s'est même manifestée parfois dans du lait de mammite nettement anormal. Rüttiman (74), qui a examiné des laits de quartiers, avance que l'influence inhibitrice exercée par la sécrétion de mammite sur le développement des bactéries n'est, pour certains micro-organismes, pas causée par la teneur en cellules accrue, mais par les modifications de la composition chimique du lait. La plus forte teneur en cellules joue, en tant que telle, un rôle à l'égard d'autres espèces de bactéries, par exemple $S$. lactis.

Keis (28) a laissé des échantillons prélevés au bidon s'acidifier spontanément et a suivi pendant 48 heures l'évolution du $\mathrm{pH}$. Il a constaté que la rapidité d'acidification ralentissait nettement entre la $10^{\mathrm{e}}$ et la $15^{\mathrm{e}}$ heure dans les échantillons à réaction CMT nettement positive. Pratiquement aucune différence ne se manifestait plus à partir de la $25^{\mathrm{e}}$ heure.

Kisza et Rotkierwicz (37) ont pu démontrer que l'activité des bactéries lactiques était nettement plus faible dans le lait de mammite subclinique. La teneur en diacétyle était aussi plus faible de 20 à 28 p. 100 dans le lait de mammite contenant 20 p. 100 de moins d'acide citrique que le lait normal. Sorokina et Slivko (83), qui ont examiné des laits de vaches prises individuellement, concluent que le développement de $S$. lactis, de $S$. cremoris, de $L$. bulgaricus et de $L$. acidophilus, mais non celui de $S$. thermophilus, est freiné dans le lait de mammite subclinique. Szakaly (86) avance que la production d'acide d'un levain de beurrerie était moins abondante et que le degré d'acidité finale obtenu était moins élevé dans le lait de mammite que dans le lait normal.

Bottazi (8) a démontré que des souches de $S$. thermophilus et de $L$. bulgaricus acidifiaient plus lentement dans un lait de mammite à 5 à 10 millions de cellules au $\mathrm{ml}$. Ce n'était pas le cas de S. lactis.

\section{Conclusion}

Le lait de mammite n'est pas un bon milieu nutritif pour certaines souches de bactéries lactiques. La forte teneur en cellules et/ou 
la composition chimique modifiée du lait en sont cause. L'éventualité pratique d'un ralentissement de l'acidification du lait de mélange, ralentissement qui résulterait de l'influence du lait de mammite, est moins clairement établie et aussi moins probable. Tout dépend naturellement du pourcentage de lait de mammite présent dans le mélange et du degré de modifications chimiques du lait.

\section{Aptitude à la coagulation du lait de mammite}

Thomé et Liljegren (88) exposent que l'aptitude à la coagulation du lait est une notion difficile à préciser et qu'elle englobe entre autres le temps de coagulation, la nature du caillé et l'aptitude à l'expulsion du sérum.

L'aptitude à la coagulation peut laisser à désirer, tant dans le cas du lait d'animaux atteints de mammite que dans celui d'animaux bien portants. Quand le lait provenant d'animaux exempts de mammite ne se coagule pas ou si sa coagulation est ralentie, la cause réside dans des influences génétiques ou des troubles du métabolisme des substances minérales.

\subsection{Temps de coagulation}

Tous les auteurs sont d'accord sur le fait que le lait de mammite peut se coaguler lentement et parfois même pas du tout, selon le degré d'inflammation du pis.

Tant Kästli et Raselli (27) que Hieteranta (20), qui ont recouru tous trois à l'épreuve de Whiteside, ont pu démontrer que le temps de coagulation moyen augmentait dans la mesure où le résultat de l'épreuve de WS était plus positif. D'après Keis (28), qui applique un CMT modifié et opère sur des échantillons prélevés au bidon, il existe un rapport statistiquement démontré entre le degré d'inflammation du pis et le temps de coagulation. En dépit du fait que le temps de coagulation moyen augmente dans la mesure où les résultats de l'épreuve de WS et du CMT modifié sont plus positifs, les auteurs précités arrivent à la constatation que les temps de coagulation individuels varient très fortement au sein d'un groupe donné. Raselli (65), qui examinait des échantillons prélevés aux quartiers, n'a pu avancer une corrélation statistiquement démontrée entre le $\mathrm{pH}$ et le temps de coagulation. Keis (28) arrive à la même conclusion à propos d'échantillons prélevés au bidon.

Hieteranta (20) a démontré que dans la plupart des cas le temps de coagulation du lait de mammite devient pratiquement égal à celui du lait normal quand on ajoute au premier des ions de Ca. Kisza et ses collaborateurs (36) estiment que la coagulation du lait n'est pas déterminée uniquement par le $\mathrm{Ca}$, mais par tout le complexe caséinecalcium et d'autres facteurs. L'addition de respectivement 10, 20, 30, 40 et 50 p. 100 de lait de mammite au lait normal a prolongé de respectivement $6,12,22,32$, et 48 p. 100 le temps de coagulation. Ces 
essais ont toutefois été effectués avec une présure fortement diluée afin de mettre les écarts bien en évidence.

\subsection{Fermeté du caillé}

On peut trouver dans la littérature des indications selon lesquelles le lait de mammite procure un caillé moins ferme. Selon Ehrlich (12), le lait de mammite caillé est peu élastique. Sommer et Matsen (81) rapportent que le lait de mammite subclinique donne un caillé de moindre fermeté. Mac Dowell (43) a observé que l'addition de seulement 20 p. 100 de lait de mammite entraîne déjà une fermeté insuffisante. Kiermeier, Renner et Djafarian (34) ont trouvé statistiquement un rapport entre la fermeté du caillé et le degré d'inflammation du pis dans des échantillons prélevés aux quartiers. Kisza et ses collaborateurs (36) arrivent aux mêmes conclusions. Kiermeier et ses collaborateurs (34) ont observé une nette diminution de fermeté du caillé consécutive à l'addition de 10 p. 100 de lait de mammite fortement modifié. Puisque les laiteries reçoivent certains mois de 20 à 30 p. 100 de lait à réaction nettement ou fortement positive au CMT, il faut bien tenir compte, selon ces auteurs, de l'influence sur la qualité fromagère du lait.

\subsection{Expulsion du sérum}

Il est rapporté ci-dessus que le temps de coagulation du lait de mammite et la consistance du caillé obtenu peuvent manifester des écarts marqués par rapport au lait normal. Ceci peut exercer une influence sur le processus d'expulsion du sérum, si important en fromagerie.

Kiermeier et Keis (31) se sont servis d'une cuve de fromagerie de laboratoire pour étudier l'influence du lait de mammite sur la fabrication du fromage, plus particulièrement sur l'expulsion du sérum. Ils ont constaté que, en faisant usage de laits de bidon, la quantité de sérum expulsée et la rapidité de cette expulsion étaient nettement moindres pour du lait de mammite que pour le lait normal ; il est apparu qu'il existe une corrélation entre ce phénomène et le degré d'inflammation du pis. L'expulsion du sérum n'a pu être suivie que pendant 1 heure, de sorte que des informations sur son évolution ultérieure font défaut. Le temps de coagulation fut aussi maintenu constant, autant pour le lait normal que pour le lait anormal. Le poids des fromages 60 minutes après le moulage était cependant plus élevé tandis que la teneur en azote du sérum expulsé était plus faible dans la mesure où le résultat du CMT modifié était plus nettement positif.

Thomé et Liljegren (88), qui ont appliqué une autre méthode pour suivre l'expulsion du sérum, méthode permettant de la suivre assez longtemps, ont obtenu des résultats quelque peu différents. Les résultats obtenus avec du lait provenant d'animaux atteints de mammite aiguë sont comparables à ceux de Kiermeier et Keis (31). 
Le lait qui présentait un sédiment après centrifugation se comportait normalement.

Sorokina (82) a pu démontrer que la formation de caillé et l'expulsion de sérum évoluent plus lentement pour le lait d'animaux atteints de mammite subclinique.

\section{Conclusion}

Le temps de coagulation du lait de mammite peut être prolongé, la fermeté de son caillé amoindrie et l'expulsion de son sérum influencée. Il faudrait des recherches plus approfondies pour examiner en quelle mesure l'addition d'ions de calcium améliorerait l'aptitude à la coagulation du lait de mammite et du lait de mélange. La moindre aptitude à la coagulation d'un lait de mélange contenant du lait normal et du lait de mammite dépendra dans une large mesure du pourcentage et de la nature du lait de mammite entrant dans le mélange.

\section{Stabilité à la chaleur}

Hammarsten (17) rapportait déjà en 1874 que la température de coagulation du lait se situe entre 130 et $150^{\circ}$ C. Kiermeier et ses collaborateurs (29) et Röse (72) commentent la littérature relative à la stabilité à la chaleur du lait normal.

Welch et Doan (92), utilisant des laits de vaches prises individuellement, pensent que le mélange de lait de mammite au lait normal nuit toujours à la stabilité, à la chaleur de ce dernier. White et Davies (93) estiment que le lait d'animaux souffrant de mammite subclinique ne présente, comparé au lait normal, ni une stabilité à la chaleur plus élevée, ni une stabilité à la chaleur moindre.

Des recherches menées par Kiermeier et ses collaborateurs (29) ont démontré que lorsque le degré d'inflammation du pis augmentait, la stabilité à la chaleur d'échantillons de lait prélevés aux quartiers diminuait fortement, de sorte que des échantillons à forte réaction positive au CMT chauffés à $130^{\circ} \mathrm{C}$ se coagulaient déjà au bout d'un temps égal au cinquième du temps de coagulation du lait normal. Les écarts entre le temps de coagulation du lait de mammite et celui du lait normal étaient moins marqués au cours d'essais effectués à $150^{\circ} \mathrm{C}$ : le facteur température y domine le facteur lait de mammite.

Feagan et ses collaborateurs (13) concluent d'une recherche que la stabilité à la chaleur du lait de quartiers de mammite subclinique est inférieure à celle du lait de quartiers non atteints. La stabilité à la chaleur d'un mélange de lait de mammite et de lait normal ne peut pas être prédite au départ de la stabilité à la chaleur des deux 
sortes de lait. La moindre stabilité à la chaleur du lait de mammite serait en corrélation avec le $\mathrm{pH}$ plus élevé.

Kiermeier et ses collaborateurs (29) ont aussi étudié l'influence de la concentration du lait normal et du lait de mammite sur la stabilité à la chaleur, ceci à des températures situées entre 110 et $150^{\circ} \mathrm{C}$. Ces recherches ont mis en évidence que le lait se coagule plus vite quand le degré de concentration augmente. Le temps de coagulation du lait de mammite concentré était plus court à une même température.

\section{Conclusion}

La stabilité à la chaleur du lait de mammite est inférieure à celle du lait normal.

\section{IV. - L'INFLUENCE DU LAIT DE MAMMITE SUR LA QUALITE DES PRODUITS LAITIERS}

La fabrication de produits laitiers de qualité exige un lait de qualité. On peut supposer que la sécrétion produite par les animaux atteints de mammite aiguë n'est pas livrée à la laiterie, mais tous les autres laits de mammite le sont. Il sera principalement question ici de l'influence de ce lait sur la qualité.

Les aspects bactériologiques du lait de mammite et l'influence possible sur la qualité feront l'objet d'un chapitre distinct.

\section{Lait de consommation}

Le lait contenant des flocons, du pus ou du sang est impropre à la consommation. Ehrlich (12) pense que, tout comme le lait colostral, le lait anormal peut causer des difficultés lors de la pasteurisation. Sous le rapport alimentaire, Kästli (25) juge que le lait de mélange contenant trop de lait de mammite est moins bon, surtout du fait de la carence en calcium. Dans les cas d'inflammation catarrhale du pis, le lait manifeste des modifications de composition fort comparables à celles du lait de vaches en fin de lactation $(25,56)$. Kästli fait état d'un goût amer lorsqu'on ne met en consommation que du lait de mammite ou un lait de mélange qui en contient une part considérable. Kiermeier et ses collaborateurs (29) avancent qu'en cas de pasteurisation, de stérilisation ou d'upérisation il n'y a pas de danger que lors du traitement de lait de mammite, il se produise une coagulation du fait de la plus faible stabilité à la chaleur. 


\section{Conclusion}

On peut et on doit être extrêmement exigeant à l'endroit du lait de consommation. On peut élever de graves objections à la présence de lait de mammite dans le lait en tant que boisson. Un effort énergique s'impose certainement en vue de limiter l'introduction de lait de mammite dans le lait de mélange.

\section{Fromage}

Les publications traitant du problème de l'influence du lait de mammite sur la qualité du fromage ont été relativement peu nombreuses ces dernières années. Quant aux publications plus anciennes, de 1931 à 1940, elles ne seront guère évoquées ici.

\subsection{Camembert}

Kiermeier et Keis $(28,32)$ ont fabriqué du Camembert à 45 p. 100 en utilisant du lait de mammite, du lait de mélange normal et du lait exempt de mammite. Le lait de mammite et le lait exempt de mammite furent sélectionnés en appliquant le CMT modifié à des échantillons de bidon. Les résultats d'une expérience sont mentionnés dans la thèse de doctorat de Keis. Le lait avait été purifié et pasteurisé et le schéma de fabrication était le même pour les trois sortes de lait. Les fromages ont été suivis pendant 5 semaines et soumis régulièrement à l'expertise organoleptique conforme aux prescriptions d'expertises allemandes. Les fromages fabriqués de lait de mammite furent rangés dans la $2^{\text {e }}$ classe de qualité ( Klasse Fein ") lors de l'expertise à une semaine, mais à 3 semaines ils ne méritaient déjà plus cette qualification. Les fromages de lait de mélange étaient aussi de $2^{\mathrm{e}}$ qualité et seuls les fromages de lait exempt de mammite étaient de qualité supérieure ( Spitzenklasse»). Les fromages fabriqués de lait de mammite avaient un goût amer, un arôme douteux et un dégagement de gaz plus ou moins marqué se manifestait après 3 semaines. On ne peut déduire de l'exposé des résultats de ces recherches si cette fermentation est inhérente au lait de mammite ou si l'on se trouve en présence d'une contamination ultérieure. La baisse de qualité des fromages fabriqués de lait de mammite est imputée à une teneur en sérum accrue, à une diminution de la teneur en extrait sec et, en partie, aussi à une augmentation de la teneur en chlorures et en protéines du sérum. Les résultats appuyant ces conclusions ne sont pas mentionnés, mais il apparaît (31) que la production au départ du lait de mammite dépassa d'environ 5 p. 100 celle au départ de lait normal. La teneur en extrait sec des fromages de lait de mammite était plus faible d'environ 3,6 p. 100 au bout d'une semaine. 


\subsection{Fromages mi-durs}

Beinert (5) a été en mesure de démontrer qu'en ajoutant 1 à 2,5 p. 100 de ferment lactique au lait de mammite lors de la fabrication de Tilsit, la qualité du fromage était réellement meilleure. Tandis que du fromage fabriqué avec du lait de mammite était de mauvaise qualité, on pouvait, en ajoutant du ferment lactique, obtenir du fromage Tilsit dont la diminution de la qualité était à peine décelable après une longue conservation.

Selon Kiermeier et Keis (32), l'influence du lait de mammite sur le Tilsit est encore plus marquée qu'elle ne l'est sur le Camembert. Du Tilsit a été fabriqué à l'échelle semi-industrielle à l'aide de lait exempt de mammite, de lait de mélange et de lait de mammite et les fromages ont été suivis pendant 140 jours. Le lait était pasteurisé avant la fabrication.

Cent dix jours après la fabrication, les fromages de lait exempt de mammite figuraient encore dans la $1^{\mathrm{e}}$ classe de la qualité ( Marken Käse »), ceux de lait de mélange dans la $2^{c}$ classe ( Klasse Fein ») tandis que les fromages de lait de mammite ne pouvaient même pas être admis dans la $3^{e}$ classe. Les défauts des fromages de lait de mammite étaient une ouverture irrégulière, des tâches de sérum, l'acidité, la fermentation, le goût désagréable.

Les données relatives au Gouda sont franchement rares. Boekhout et De Vries (6) ont affirmé qu'ils pouvaient fabriquer un Gouda normal au départ de lait de mammite additionné de $1 / 3$ de lait normal. Schipper (75) communique qu'il a été observé au cours d'une étude de l'influence du lait de mammite sur les techniques de transformation, que le temps de coagulation peut être prolongé en cas de pourcentages élevés de lait de mammite et que le caillé présente alors une structure anormalement molle et a tendance à retenir beaucoup de liquide. Aucune influence n'a toutefois été observée au cours d'autres essais.

\subsection{Fromages à pâte dure}

Il ressort du tour d'horizon fait par Mattick et ses collaborateurs (49) qu'un certain nombre de recherches effectuées avant 1937 en Grande-Bretagne, aux Etats-Unis et en Nouvelle-Zélande indiquent que le lait de mammite peut causer des difficultés dans la fabrication du Cheddar.

Mac Dowell (43) a fabriqué du fromage Cheddar de lait provenant des mêmes vaches, aussi bien de quartiers sains que de quartiers pour lesquels un test au bleu de bromotymol était positif. Lorsque, dans la fabrication, on utilisait du lait pasteurisé et procédait selon la même technique de fabrication, on ne constatait pas de différence dans la qualité. Le fromage provenant du lait de mammite contenait plus de sérum que le fromage témoin et la teneur en matières grasses était légèrement plus faible, ceci à cause de la perte 
plus élevée de matières grasses dans le sérum. Le rendement plus bas avait pour cause la teneur en caséine plus faible du lait de mammite. Lorsqu'on utilisait du lait pasteurisé, l'addition de 25 p. 100 de lait de mammite au lait normal n'avait pas d'influence sur la qualité, la composition et le rendement du fromage.

Hill et ses collaborateurs (21) ont pu démontrer que le défaut «soft-curd» se manifestait dans le Cheddar fabriqué d'un lait dont la composition était très proche de celle du lait de mammite subclinique. On pourrait remédier à ce défaut par une addition d'ions de $\mathrm{Ca}$ et de $\mathrm{P}$ et en recourant à une technique de fabrication adaptée. Mattick et ses collaborateurs (48) signalent qu'une infection latente par $S$. agalactiae rend le lait impropre à la fabrication de Cheddar : tant la qualité que le rendement étaient inférieurs. Johns et ses collaborateurs (24) signalent qu'à une exception près, un lait de ce genre a donné un fromage comparable au fromage de lait normal. D'autre part, on rencontre chez Marquardt et Hucker (47) l'opinion que l'on peut obtenir une même qualité et un même rendement quand on utilise de grandes quantités de ferment lactique ou que l'on ajoute de l'acide à ce lait.

L'influence du lait de mammite sur l'Emmental n'a guère fait l'objet d'examens systématiques. Kästli (25) pense que l'inclusion d'agglomérats de cellules, de pus, etc. peut provoquer une fermentation anormale et une dégradation de protéines anormale en ces endroits du fromage. De tels cas sont toutefois plutôt rares, puisque ces agglomérats de cellules et de pus restent dans la cuve sous la forme de poussière de caillé. Cet auteur estime en outre que la diminution de la teneur en lactose ne peut entraîner que dans des cas extrêmes une diminution de l'aptitude à l'acidification du lait. Le rendement plus médiocre en fromage Emmental n'est pas causé uniquement par la diminution de l'aptitude à la coagulation, mais aussi par la plus faible teneur en caséine.

Delambre (11) relève que la fabrication d'Emmental en HauteSavoie se heurte parfois à des difficultés du fait de ce qu'on appelle les "fausses places ». Il pense que le lait de mammite joue un rôle en l'occurence. Mougin (51) rapporte que les fromages de Gruyère et l'Emmental fabriqués de lait de mammite manifestent une tendance à retenir trop de sérum et à sécher difficilement.

\section{Conclusion}

L'influence du lait de mammite sur la qualité du fromage est un point de discussion et est en rapport avec le type de fromage, la nature du lait de mammite et la technique de fabrication. Une partie de ce que nous savons jusqu'ici est basée sur l'expérience pratique et non sur la recherche systématique. Peu de publications récentes traitent de l'influence du lait de mammite sur la qualité du fromage 
et les résultats connus ne sont basés que sur un nombre restreint de fabrications. De ces expériences il ressort qu'il faut tenir compte d'une diminution possible de la qualité du fromage.

La question qui se pose et qui devrait être résolue est la suivante :

Est-il possible de fabriquer avec du lait de mammite du fromage de bonne qualité en faisant éventuellement usage de $\mathrm{CaCl}_{2}$ et d'un schéma de fabrication adapté ?

Des recherches plus vastes et plus approfondies sont souhaitables. Il faut, en outre, tenir compte du fait que le lait de mammite employé dans la pratique fromagère n'est pas traité tel quel, mais sous la forme de lait de mélange. Des recherches sur l'influence que l'addition de lait de mammite exerce sur la qualité sont aussi souhaitables. Cependant, la pratique en Belgique et aux Pays-Bas montre qu'on n'éprouve pas de difficultés en fabriquant du fromage Gouda et Cheddar à partir de lait de mélange contenant du lait de mammite. La médiocre aptitude à la coagulation du lait de mammite étant par excellence le facteur susceptible d'influencer la qualité du fromage, il semble indiqué de rechercher pour certains types de fromages en quelle mesure l'addition d'ions de $\mathrm{Ca}$, éventuellement combinée avec des modifications de la technique de fabrication peut y remédier.

\section{Beurre}

Schneck et Dörwald (76) ont transformé en beurre une crème provenant d'un lait de mammite ne présentant aucune altération macroscopique. La teneur en diacétyle de ce beurre était médiocre.

Kiermeier et Keis $(28,32)$ ont obtenu un beurre de mauvaise qualité en partant de crème de lait de mammite. Une seule série d'essais a été mise en œuvre avec 2000 litres de lait normal, de lait exempt de mammite et de lait de mammite ainsi qu'avec un ferment lactique normal, tandis que le ferment lactique employé pour une autre série d'essais fut cultivé dans un lait de mammite fortement positif. Le beurre ainsi fabriqué fut entreposé à $10^{\circ} \mathrm{C}$.

Dans le cas où l'on avait employé le ferment lactique normal, le beurre fabriqué de crème de mélange et de crème exempte de mammite fut reconnu, dix jours après la fabrication, être de $1^{\mathrm{e}}$ classe, le beurre de crème de lait de mammite n'obtenant que la qualification de $2^{\text {e }}$ classe. Après 30 jours, le beurre de lait de mélange et de lait exempt de mammite fut rangé dans la $2^{e}$ classe et le beurre de lait de mammite fut qualifié de $3^{\text {e }}$ classe.

Dans le second essai, où l'on avait employé un ferment anormal, le beurre de lait de mammite fut reconnu de $2^{\text {e }}$ qualité à 10 jours et de $3^{\text {e }}$ qualité à 30 jours. Les deux autres beurres passèrent de la $1^{\mathrm{e}}$ qualité, à 10 jours, à la $2^{\mathrm{e}}$ qualité, à 30 jours. L'examen des échantillons de beurre se limitait, en principe, aux expertises orga- 
noleptiques. Il est toutefois mentionné que la plus forte teneur en protéines manifestée par le lait de mammite n'exerçait aucune influence sur la teneur en protéines du beurre.

Szakaly (85) signale que, comparée à la crème normale, la crème de lait de mammite s'acidifiait plus lentement et exigeait un barattage prolongé de 30 à 37 p. 100 . Le beurre de lait de mammite était de moins bonne qualité et des défauts d'odeur et de goût ne tardaient pas à se manifester pendant la conservation.

Brus et ses collaborateurs (9) ont présenté un rapport sur la qualité de beurres fabriqués de laits à fortes et à faibles teneurs en cellules. Le lait normal et le lait de mammite furent sélectionnés en utilisant le BMR sur des échantillons de bidon. Ils procédèrent quatre fois, à l'échelle semi-pratique, à une fabrication de beurre en utilisant du lait normal et du lait de mammite. Aucune différence ne fut observée entre les lots pendant 2 mois de conservation à $-12^{\circ} \mathrm{C}$. Au cours d'une conservation de plus longue durée, le beurre de lait de mammite se révéla de plus mauvaise qualité, ce qui se traduisait par des phénomènes d'oxydation. La teneur en cuivre du beurre de mammite était plus élevée que celle du beurre témoin, ceci en dépit des mesures prises à la ferme pour exclure la contamination par le cuivre. Les auteurs émettent l'hypothèse d'un enrichissement préférentiel en cuivre du beurre fabriqué de lait de mammite et du rôle possible de certains enzymes.

Kästli (25) pense que, d'une manière générale, le lait de mammite ne nuit guère à la qualité du beurre. Il ne faut s'attendre à un rendement moindre que dans les cas d'addition de pourcentages extrêmement élevés de lait de mammite. Dans ces cas, la teneur en diacétyle est également influencée par la moindre teneur en acide citrique du lait.

\section{Conclusion}

Quoique les recherches de date récente soient limitées et ne portent que sur un petit nombre de fabrication d'essai, il existe des indices que l'emploi de crème de lait de mammite peut entraîner une baisse de la qualité du beurre. Des recherches plus approfondies sont certainement souhaitables. Tenant compte du fait que la pratique beurrière n'emploie que du lait de mélange et pas de lait de mammite, une influence sur la qualité nous semble moins probable quand on emploie de la crème pasteurisée. Des recherches plus vastes sont souhaitables.

\section{Produits laitiers acides}

La littérature qui traite de l'influence du lait de mammite sur les produits laitiers acides est très peu abondante. Schott (77) a examiné si le lait de mammite subclinique influence la qualité du 
yogourt. Il est apparu que ce n'est pas le cas. Une coloration jaunâtre du yogourt était la plus nette dans le lait de mammite pur. L'addition de lait de mammite jusqu'à raison de 100 p. 100 n'exerça pas d'influence défavorable et ne pouvait être statistiquement démontrée sur le nombre de germes, l'évolution de l'acidification et le rapport bâtonnets/coques.

\section{Lait condensé et poudre de lait}

Welch et Doan (92) ont démontré que le lait concentré provenant d'animaux atteints de mammite subclinique était bien moins thermostable que le lait normal condensé. Rogers, Deysher et Evans (70), Holm, Webb et Deysher (22) n'ont relevé aucun rapport entre la stabilité à la chaleur du lait et celle du lait concentré.

Kiermeier et ses collaborateurs (29) signalent que dans la fabrication de lait condensé à un rapport d'évaporation $3 / 1$ et à une température de $110-120^{\circ} \mathrm{C}$ maintenue pendant 15 à 20 minutes, l'emploi de lait de quartiers à réaction fortement positive au CMT présente le clanger de coagulation. Des essais de chauffage ont prouvé qu'un lait de ce genre se coagule déjà au bout de 17,7 minutes à $110^{\circ} \mathrm{C}$ et déjà au bout de 7,6 minutes à $120^{\circ} \mathrm{C}$. Les mêmes auteurs ont pu démontrer que la limite de stabilité est atteinte, pour un rapport d'évaporation de $3 / 1$ et une combinaison de température et de durée d'échauffement de 15 minutes à $120^{\circ} \mathrm{C}$, lorsque le lait de mélange contient 50 p. 100 de lait fortement positif. Kiermeier et ses collaborateurs (29) estiment que chaque laiterie qui fabrique du lait condensé devrait connaître assez exactement le pourcentage de lait à forte teneur en cellules dont elle prend livraison.

Feagan et ses collaborateurs (14) avancent, eux aussi, que le pourcentage de lait de collecte provenant d'animaux atteints de mammite subclinique est un facteur d'une réelle importance, qui ne détermine pas seulement la stabilité à la chaleur du lait, mais aussi l'effet que peut avoir, par exemple, d'addition de sels stabilisants, tant dans la fabrication directe de lait condensé que dans celle de la poudre de lait destinée à la reconstitution du lait condensé.

Quant à la poudre de lait, ces auteurs affirment qu'il n'existe aucun rapport entre la stabilité à la chaleur du lait et celle de la poudre de lait reconstitué qui en est fabriquée.

\section{Conclusion}

Le lait de mammite joue aussi un rôle dans le problème complexe de la stabilité à la chaleur du lait et de l'influence de cette stabilité sur la fabrication de lait concentré. Des recherches plus approfondies sont certainement souhaitables. 


\section{V. - LES BACTERIES DU LAIT DE MAMMITE ET LEUR INFLUENCE SUR LA QUALITE HYGIENIQUE DES PRODUITS LAITIERS}

Il convient de préciser avant tout que lorsque le lait est chauffé à la température de pasteurisation ou au-delà, celle-ci tue toutes les bactéries qui menacent la santé de l'homme ou créent des difficultés. Il faut néanmoins tenir compte de la présence possible dans un tel lait ou dans les produits laitiers fabriqués de ce lait d'entérotoxines produites par des staphylocoques ou de toxines sécrétées par certaines souches de bactéries coliformes. Kästli (25) remarque toutefois que les staphylocoques producteurs d'entérotoxines présents dans le lait ne proviennent qu'exceptionnellement d'un pis infecté, mais sont généralement introduits dans le lait par une personne contaminée. Worseck (94) affirme, par contre que 67 p. 100 des staphylocoques de mammite sont des producteurs d'entérotoxines. Tant les bactéries coliformes que les staphylocoques peuvent se multiplier très rapidement dans le lait non réfrigéré.

Les bactéries présentes dans le lait de mammite peuvent se retrouver dans les produits lorsqu'on omet de pasteuriser le lait.

Les mammites à bactéries coli-aérogènes sont très dangereuses pour la fabrication de fromage de lait cru parce qu'elles provoquent le défaut de "fermentation prématurée ». Selon Kästli (25), les mammites à streptocoques provoquées par S. pyogenes et $S$. epidermicus sont rares, mais très dangereuses pour la santé. Ce même auteur (25) est d'avis que les mammites imputables aux salmonelles, aux pneumocoques, au $\mathrm{Cl}$. perfringens, aux pasteurelles, etc., qui se manifestent généralement sous une forme aiguë et sont pathogènes pour l'homme, sont très rares. Moller-Madsen et ses collaborateurs (50) signalent qu'il a été impossible, 10 jours après la fabrication, de retrouver $S$. pyogenes dans des fromages danois Samsö et Havarti fabriqués de lait pasteurisé réinfecté par ces bactéries. Moller-Madsen et ses collaborateurs (50) ont pu démontrer que dans les fromages Samsö et Havarti fabriqués de lait réinfecté par des salmonelles et des staphylocoques, ces bactéries ne meurent pas assez vite pour que ces fromages en soient exempts au moment où ils sont mûrs pour la consommation. Reiter et ses collaborateurs (68) ont pu prouver que les staphylocoques peuvent se multiplier dans le caillé pendant la fabrication.

Kästli (25) fait aussi état de la possibilité que des bactéries pathogènes pour l'homme présentes dans le lait de mammite non pasteurisé peuvent se retrouver dans le beurre. 


\section{VI. - CONCLUSION GENERALE}

La littérature procure de nombreuses données sur la différence de composition entre le lait de mammite et le lait normal. Les observations faites ne concordent pas toujours. Ceci n'a rien d'étonnant, étant donné que le lait de mammite peut dévier de peu jusqu'à très fort du lait normal, et que l'on applique des critères différents pour déterminer si l'on se trouve ou non en présence de lait de mammite. De plus, les déterminations de certaines substances ne sont pas toujours faites par les mêmes méthodes. Certaines anomalies que l'on peut relever dans les échantillons prélevés aux quartiers ne peuvent se retrouver que difficilement ou pas du tout dans le lait d'animaux pris individuellement et dans le lait de bidon.

En ce qui concerne la transformation de lait de mammite en produits laitiers, il convient de souligner que, malgré la relative rareté de recherches récentes, il faut tout de même tenir compte d'une possible influence nuisible à la qualité de certains produits laitiers. Un complément de recherches est fort souhaitable.

Quant à la mise en œuvre de lait de mélange contenant des quantités pas trop considérables de lait de mammite, on peut penser qu'elle ne suscitera généralement pas ou presque pas de problèmes. Tout dépend naturellement du pourcentage et de la nature du lait de mammite. Il serait souhaitable de disposer de données plus précises relatives au pourcentage de lait de mammite qui peut être ajouté au lait de mélange sans que cela ait une influence sur la qualité des produits laitiers.

Le lait de mélange livré à nos laiteries comprend un certain pourcentage de lait de mammite et ce pourcentage peut être parfois relativement élevé. Cet état de choses se maintiendra sans doute encore pas mal de temps. La tâche actuelle de la technologie laitière consiste à limiter dans toute la mesure du possible l'influence éventuelle du lait de mammite sur la qualité des produits laitiers. Ce n'est évidemment qu'une solution de fortune. La vraie solution consiste à lutter par tous les moyens contre la mammite et les problèmes qu'elle pose.

\section{S u m m a ry}

The aim of this study was to collect bibliographical data concerning the influence of mastitis on milk composition and in connection with production and quality of dairy products.

Although the presence of antibiotics in mastitis milk, due to intense use in controlling the disease is a very important aspect of the problem, it is not discussed here. 
Disturbances in the conversion of blood components into normal milk components seems to be generally accepted.

The opinions on the influence of udder inflammation on milk composition are sometimes rather divergent. This is not surprising as mastitis milk can differ from normal milk in a lesser up to a higher extent. Besides, various criteria are used for the appreciation of mastitis milk. Moreover, determinations of certain substances have not always been executed by the same methods.

Mastitis milk is not a good culture medium for certain lactic acid bacterial strains for reason of its high cell content and/or the altered chemical composition of the milk.

In cheese manufacture the coagulation time can be lengthened, the firmness of the curd can be diminished and the whey-running can be influenced.

Mastitis milk has a lesser heat stability than normal milk.

We may accept that the udder-secretion from animals suffering from acute mastitis is not delivered to the dairies. All other mastitis milk however is delivered. Il is mainly the influence of this milk on the quality which will be discussed.

High demands may and must be made upon marketing milk. A great effort must certainly be made in order to limit the admixture of mastitis milk.

The influence of mastitis milk on the quality of cheese is connected to the nature of the milk used, the cheese type and the technique employed in manufacture. Our present knowledge is partially based on experience and not on research. In recent years little has been published concerning the influence of mastitis milk on the quality of cheese and the results are based on a restraint number of trials only. From these researches it appears that a possible diminution of quality must be taken into account.

The slower coagulation with rennet of mastitis milk is the factor which influences mostly the quality of the cheese, therefore it seems to be advisable that research workers should look out for a modified manufacturing technique for some types of cheese, to compensate for this.

Even though recent research is limited and only a small number of experimental trials is discussed, there are indications that the quality of butter, manufactured from mastitis cream may be lowered.

Mastitis milk may interfere in the rather difficult problem of heat stability of milk and influence the manufacture of evaporated milk. There are indications that mastitis milk causes difficulties in the preparation of evap.

How bulk milk, containing mastitis milk, will react in the manufacture of dairy products depends on the percentage of mastitis milk and the nature of the chemical alteration of this milk. 
It can be taken for granted that when milk is heated to normal pasteurisation temperature or higher, the bacteria which are pathogenic to humans or which create difficulties in the manufacture of dairy products are killed. If not thus heat-treated, then the bacteria in the mastitis milk get into these products and can give rise to difficulties in the manufacture as well as causing illnesses in man.

As conclusion we must point out that as far as the manufacture of dairy products from mastitis milk is concerned, even though relatively little research has recently been carried out, account must however be taken of a detrimental influence on the quality.

If not too large a quantity of mastitis milk is added, it may be assumed that no problems, or at any rate only very few problems, will arise in manufacturing bulk milk.

More research about the influence of mastitis milk on the technological practicability of the milk and on the quality of the dairy products is strongly desirable.

\section{VII. - Littérature}

[1] Alexander (W. H.) et Leech (F. B.). - J. Dairy Res., 27, 1960, 19.

[2] Arima (S.). - Mem. Fac. Agric. Hokkaïdo Univ., 4, 1962, 34, réf. D. Sci. Abstr., 25, 1963, 121.

[3] Ashworth (U. S.) et Blosser (Th.). - J. Dairy Sci., 47, 1964, 696.

[4] Baumgartner (H.) et Walser (R.). - Schweiz. Milchztg, 85, 1959 ; Wiss. Beil. $\mathrm{Nr} 63$.

[5] Beinert (B.). - Thèse de doctorat, Kiel, 1941.

[6] Boekhout (F. W.) et DE VRIEs O. - Verslag Landbouwkundig Onderzoek, 12, 1912, 139.

[7] Bortree (A. L.), Caroll (E. J.) et Schalm (O. W.). - J. Dairy Sci., 45, 1962, 1465.

[8] Bottazi (V.). - D. Sci. Abstr., 30, 1968, 989.

[9] Brus (D. H. J.), JaArtsveld (F. H. J)., Van Werven (E.) et Bannenburg (H. J.). Nederl. Melk-en Zuiveltijdschrift, 20, 1966, 37.

[10] Davis (J. G.) et Mc Clemont (J.). - J. Dairy Res., 10, 1939, 94.

[11] Delambre (Cl.). - Thèse de doctorat, 1962, Ecole Nationale Vétérinaire de Lyon.

[12] Ehrlich (C.). - Dte Molk.-Ztg., 53, 1939, 1552.

[13] Feagan (J. T.), Griffin (A. T.) et Lloyd (G. T.). - J. Dairy Sci., 49, 1966, 933.

[14] Feagan (J. T.), Griffin (A. T.) et Lloyd (G. T.). - J. Dairy Sci., 49, 1966, 940.

[15] Filipovith (D.) et Filipovitch (D.). - Lait, 36, 1956, 608.

[16] Guthrie (E. S.) et Herrington (B. L.). - J. Dairy Sci., 43, 1960, 843.

[17] Hammarsten (0.). - Jahresbericht über die Fortschritt der Tierchemie, 4, $1874,135$.

[18] HaUKe (H.). - Milchwissenschaft, 21, 1966, 700.

[19] Heeschen (W.). - Int. Dairy Congress, 1966, A, 391.

[20] Hieteranta (M.). - Int. Dairy Congress, 1962, B, 557.

[21] Hill (R. D.), Laing (R.R.), Snows (N.S.) et Hammond (L. A.). - Aust. J. Dairy Technol., 20, 1965, 122. 
[22] Holm (G. E.), Webb (B. H.) et Deyscher (E. F.). - J. Dairy Sci., 15, 1932, 331.

[23] Imamura (T.), KadaoKa (K.), Ishil (R.). - Jap. J. zootech. Sci., 35, 1964, 117 réf. D. Sci. Abstr., 26, 1964, 601.

[24] Johns (C. K.), Hickx (T. J.) et Gibson (C. A.). - J. Dairy Res., 11, 1940, 298.

[25] Kastli (P.). - Milchkunde II. Buchverlag Verbandsdrukerei A.G., Bern 1963.

[26] Kastli (P.) et Graber (H.). - Schweiz. Ztschr. Path. Bakt., 18, 1955, 1006.

[27] Kastli (P.) et Raselli (R.). - Int. Dairy Congress 1959, 1, 128.

[28] KeIs (K). - Thèse de doctorat, 1963, Techn. Hochsch. München.

[29] Kiermeier (F.), Djarfarian (M.) et Kirchmeier (O.). - Z. Lebensmittelunters. u. -Forsch. 133, 1967, 208.

[30] Kiermeier (F.) et Graf zu Solm-Baruth (H.) - Milchwissenschaft, 21, 1966, 441.

[31] Kiermeier (F.) et KeIs (K). - Milchwissenschaft, 19, 1964, 79.

[32] KiermeieR (F.) et KeIS (K.). - Z. Lebensmittelunters. u. -Forsch., 124, 1964, 184.

[33] Kiermeier (F.) et Keis (K.). - Milchwissenschaft, 20, 1965, 663.

[34] Kiermeier (F.), Renner (E.) et Djafarian (M.). - Z. Lebensmittelunters. $u$. -Forsch., 132, 1967, 352.

[35] Kisza (J.), Karwowicz (E.) et Sobina (A.). - Milchwissenschaft, 19, 1964, 437.

[36] Kisza (J.), KRUK (A.) et RotKIEwicz (W.). - Milchwissenschaft, 22, 1967, 558.

[37] Kisza (J.) et RotKiEwicz (W.). - Michwissenschaft, 22, 1967, 627.

[38] Kisza (J.) et Sobina (A.). - Milchwissenschaft, 17, 1962, 544.

[39] Kisza (J.) et Sobina (A.). - Milchwissenschaft, 18, 1963, 171.

[40] Lecce (J. C.) et Legates (J.). - J. Dairy Sci., 42, 1959, 698.

[41] LERCHE (M.). - Lehrbuch der tierärztlichen Milchiiberwachung, Verlag Paul Parey, Berlin und Hamburg, 1966.

[42] Luedecke (L. O.). - J. Dairy Sci., 47, 1964, 696.

[43] Mc Dowell (F. H.). - N.Z. Jl. Sci. and Technol., 27, 1946, 480.

[44] Marmori (M. G.), Nani (S.) et Redaelli (G.). - Atti Soc. ital. Sci. vet., 11, 1957, 804, réf. D. Sci. Abstr., 20, 1958, 779.

[45] Marmori (M. G.) et Redaelli (G.). - Archo vet. ital., 10, 1959, 545, réf. D. Sci. Abstr., 22, 1960, 205.

[46] Marquardt (R. R.) et Forster (T. L.). - J. Dairy Sci., 45, 1962, 653.

[47] Marquardt (J. C.) et Hucker (G. J.). - Techn. Bull. N.Y. St. agric. Exp. Stat. 242.

[48] Mattick (A. T. R.), Davies (W. L.) et Dearden (D. V.). - XI e Int. Dairy Congress, Berlin, 1937, 1, 283.

[49] Mattick (A. T. R.), Hiscox (E. R.) et Davis (J. G.). - J. Dairy Res., 8, 1937, 390.

[50] Moller-Madsen (A.), Petersen (A. H.) et Bruhn (P, A.). - Milchwissenschaft, $15,1960,403$.

[51] Mougin (N.). - Techn. Lait, 15, 1963, 15.

[52] Müllen (J. E. C.). - J. Dairy Res., 17, 1950, 288.

[53] Nani (S.) et Defranceschi (L.). - Archo Vet. ital., 8, 1957, 17, réf. D. Sci. Abstr., 19, 1957, 857.

[54] Nani (S.) et Redaelli (G.). - Atti Soc. ital. Sci. vet., 11, 1957, 799, réf. D. Sci. Abstr., 20, 1958, 778.

[55] Natzke (R. P.), Schultz (L. H.), Barr (G. R.) et Holtmann (W. B.). - J. Dairy Sci., 48, 1965, 1295.

[56] NeugschwendtNeR (St.). - Milchwirtsch. Forschung, 5, 1928, 1.

[57] Nicolet (J.). - Milchwissenschaft, 17, 1962, 307-362.

[58] Nilsson (G.). - Int. Dairy Congress, 1956, 3, 330.

[59] Nilsson (G.). - K. Lantbr. Högsk. Ann., 23, 1957, 73-122, réf. D. Sci. Abstr., $20,1958,246$. 
[60] Oberosler (R.), Aguggini (G.), Bergamaschi (M.), Chiesa (F.) et Perini (G.). Archo vet. ital., 12, 1961, 145, réf. D. Sci. Abstr., 23, 1961, 389.

[61] OBRiger (G.). - Milchwissenschaft, 17, 1962, 550.

[62] O'Donovan (J.), Dodd (F. H.) et Neave (F. K.). - J. Dairy Res., 27, 1960, 115.

[63] OWEN (E. C.) : Some aspects of the métabolisme of vitamine A and carotene, W.R.N.D., 5, 1965.

[64] Pette (J. W.). - Misset's Zuivel, 69, 1963, 1041-1 123.

[65] Raselli (R.). - Thèse de doctorat, 1958, Université, Bern.

[66] Redaelli (G.) et Nani (S.). - Atti Soc, ital. Sci. vet., 11, 1957, 801, réf. D. Sci. Abstr., 20, 1958, 779.

[67] Reimund. - Molk. Ztg, 43, 1929, 2133.

[68] Reiter (B.), Fewins (B. G.), Fryer (T. F.) et Sharp (E. M.). - J. Dairy Res., $31,1964,261$.

[69] Renk (W.) et Teute (H. W.). - Berl. und Münchl. tierärztl. Wschr., 71, 1958, 23.

[70] Rogers (L. A.), Deysher (E. F.) et Evans (F. R.). - J. Dairy Sci., 4, 1921294.

[71] Rook (J.A.F.). - D. Sci. Abstr., 23, 1961, 251-303.

[72] Röse (D.). - D. Sci. Abst., 25, 1963, 45.

[73] Rowland (S. F.), Neave (F. K.), Dodd (F. H.) et Oliver (J.). - XV Int. Dairy Congress, 1959, 1, 121.

[74] Rüttiman (G.). - Thèse de doctorat, 1956, Université Bern.

[75] Schipper (C. J.). - Officieel Org. K. Ned. Zuivelbond, 60, 1968, 658.

[76] SCHNecK (A.) et Dörwald (W.). - Vorratspfl. und Lebensmitt. Forsch., 1, $1938,676$.

[77] Sснотт (G.). - Thèse de doctorat, 1966, Univ. München.

[78] Seelemann (M.). - Molk. - u. Käs.-Ztg, 6, 1955, 1817.

[79] Seelemann (M.). - Milchwissenschaft, 18, 1963, 401.

[80] Seelemann (M.) et Obriger (G.). - Kieler Milchw. Forsch. Ber., 8, 1956, 23.

[81] Sommer (H. H.) et Matsen (H.). - J. Dairy Sci, 18, 1935, 741.

[82] Sorokina (O. F.). - D. Sci. Abstr., 30, 1968, 972.

[83] Sorokina (O. F.) et Slivko (V. V.). - D. Sci. Abstr., 28, 1966, 215.

[84] STOCKER (W.). - Allgemeine Grundlagen der Käsereitechnik, Volkwirtschaftlicher Verlag G.m.b.H. Kempten (Allgaü), 1957.

[85] Szakaly (S.). - D. Sci. Abstr., 27, 1965, 2377.

[86] Szakaly (S.). - D. Sci. Abstr., 30, 1968, 2067.

[87] Teute (H.W.). - Berl. und Münchl. tierärz. Wschr., 74, 1961, 185.

[88] Thomé (K. E.) et LilJegren (G.). - Int. Dairy Congress, 1959, III, 1922.

[89] Waite (R.) et Blackburn (P. S.). - J. Dairy Res., 24, 1957, 328.

[90] Waite (R.) et Blackburn (P. S.). - J. Dairy Res., 30, 1963, 23.

[91] Wartenberg (L.). - Med. vet. Warz., 12, 1956, 422, réf. D. Sci. Abstr., 19, $1957,414$.

[92] Welch (R. C.) et Doan (F. J.). - J. Dairy Sci., 18, 1935, 287.

[93] White (J. C. D.) et Davies (D. T.). - J. Dairy Res., 25, 1958, 281.

[94] WoRsecK (M.). - Arch. Lebensmittelhyg., 7, 1956, 145.

Reçu pour publication en décembre 1968. 